\title{
An external millisecond timer for the Apple Macintosh with applications to cross-modal lexical priming
}

\author{
SARAH C. WAYLAND \\ Northeastern University, Boston, Massachusetts \\ and \\ ALAN K. THOMPSON \\ Massachusetts Institute of Technology, Cambridge, Massachusetts
}

\begin{abstract}
The circuit and program described in this report allow researchers to use Macintosh personal computers to conduct research that requires timing with millisecond accuracy. This is accomplished with external response keys and an external clock that sends characters through the Macintosh's serial port. For researchers interested in cross-modal lexical priming tasks, a tachistoscopic slide shutter is incorporated to allow for accurately timed presentation of visual stimuli. Because the visual display and response keys are external to the computer, display and reaction times are not subject to the timing constraints inherent to the keyboard or the Macintosh Event Manager. All circuit diagrams and code are in the public domain.
\end{abstract}

Many laboratories are equipped with Apple Macintosh computers that run data analysis, word processing, and graphics applications. These computers can also present stimuli and collect data. The Macintosh can be particularly challenging, however, for those who need millisecond accuracy in recording reaction times, especially if subjects respond by using an Apple Desktop Bus (ADB) device. The difficulty lies in the fact that the Macintosh Event Manager reports the time of an event (a keypress, mouse click, etc.) to the nearest tick $(16.67 \mathrm{msec})$. By circumventing the Event Manager, you may be able to report the event to within 10 or $11 \mathrm{msec}$ for ADB devices, or within approximately $3 \mathrm{msec}$ for MacPlus and lower keyboards (for more information, see Inside Macintosh, Vol. 1, chap. 8). This solution, however, is highly machine- and operating-system-dependent. Another difficulty lies in the fact that the Macintosh operating system does not allow the user to easily stop all system activities while making

This work was supported by a 1990 APA dissertation award to S.W., then at Brandeis University. All correspondence should be sent to S. Wayland, now at the Department of Psychology, 125 Nightingale Hall, Northeastern University, Boston, MA 02115 (e-mail: wayland @northeastern.edu). Alan Thompson is now at the Department of Physics, Harvard University, Cambridge, MA 02138 (e-mail: akt @hussle.harvard.edu). Users who have access to ftp (contact your computer system administrator) can obtain copies of the circuit diagram. Circuit diagrams and a more extensive example of the code at the ftp site: thor.acc.stolaf.edu (IP\# 130.71.192.1) in pub/macpsych. Log in as anonymous, and use your username as the password. Copies of the software can also be obtained by sending a blank 3.5 -in. floppy disk along with a self-addressed, stamped envelope (with enough postage for returning the floppy disk) to the first author at the above address. a measurement. This leads to an error in measurement that may be unacceptable for some applications (but see Ulrich \& Giray, 1989). Researchers have proposed numerous solutions to this problem, including synchronizing the visual information on the screen with the onset of timing routines (Resnik, 1990) and other ways to access the clock in the Macintosh computer while correcting for the system activities that may occur during a timing sequence (e.g., Westall, Perkey, \& Chute, 1986, 1989).

This paper presents a solution to the timing problem which is also useful for researchers interested in running cross-modal lexical priming tasks (see, e.g., Swinney, 1979) on a Macintosh computer. The cross-modal lexical priming task involves presenting an auditory stimulus to a subject and, after some accurately determined time interval, presenting a visual stimulus to which the subject responds. Although the circuit described here has been used primarily for cross-modal lexical decision tasks, it is also designed to be used as an external timing device that can monitor reaction times to stimuli displayed on the Macintosh (see Implementation, below). By removing the timing operation from the Macintosh itself, this system allows for true millisecond accuracy in measurement of reaction times. It should also be noted that reaction times are measured via external reaction keys. This removes the uncertainty involved with responding via $\mathrm{ADB}$ devices (e.g., the mouse and keyboard). Other external timers, such as the Strawberry Tree, GW Instruments, and other data acquisition boards, can be used for the same purpose. If the user is on a budget, however, our solution can be implemented for under $\$ 50$ in 1991 U.S. dollars. In addition, as described in the section discussing the system monitoring circuit, up to six different responses can be recorded. 


\section{THE SYSTEM}

Figure 1 depicts the overall setup for doing a crossmodal lexical priming experiment. Auditory stimuli are presented to the subject through headphones that monitor only one channel of the tape player. This channel usually contains sentences or words (the auditory stimuli). The other channel of the tape player is fed into the discriminator, which monitors the output for a signal, which is usually a short (e.g., $50-\mathrm{msec})$ square wave tone. The discriminator can be adjusted to respond only to signals above a certain decibel level. This feature is useful for dealing with spurious noise that can find its way onto tapes and computer editing systems. Once the discriminator has detected the presence of a signal, it presents the visual stimulus by opening a tachistoscopic shutter ${ }^{1}$ that is mounted on a slide projector and simultaneously signals the System Monitor Circuit to begin sending characters that indicate that the visual stimulus has been presented (" $Q$ " is the character used for this purpose). The tachistoscopic shutter on the slide projector is open for $50 \mathrm{msec}^{2}$ When the subject presses one of the response keys, the character sending circuit sends different sets of characters, depending on which key has been hit (" $R$ " or " $T$ "'). Once the key is released, the circuit resets itself and sends the default character ("P"). Users who are interested only in recording reaction times need only remove the shutter driver portion of the setup (and circuit), leaving the one output from the discriminator circuit to the timing portion of the circuit.

The characters are sent to the Macintosh computer through the serial (modem) port, which accepts data at roughly 960 characters per second $(9,600$ baud). Independent measurements of the number of characters our Macintosh Plus received in $10 \mathrm{sec}$ indicated that the actual baud rate for our setup was $9,700 \pm 20$ bytes, or $970 \pm 2$ characters per second. ${ }^{3}$ Thus, reaction times counted from the onset of the triggering tone could be measured to an accuracy of $1.03 \mathrm{msec}$ with our Macintosh Plus. We highly recommend that baud rate be measured for each system, because the conversion may be slightly different for different computers. Please also beware that because the response time on the tachistoscopic shutter we used is $30 \pm 2 \mathrm{msec}$, reaction times to visually presented stimuli are accurate to within $\pm 3 \mathrm{msec}$. This is acceptable for the $20-40 \mathrm{msec}$ reaction time differences usually found in cross-modal lexical priming.

In this configuration, the Macintosh is used only to collect information-the tape player and slide projector present the stimuli. This was done to avoid the timing problems associated with using multiple events on the Macintosh. The computer is thus in a data collection mode

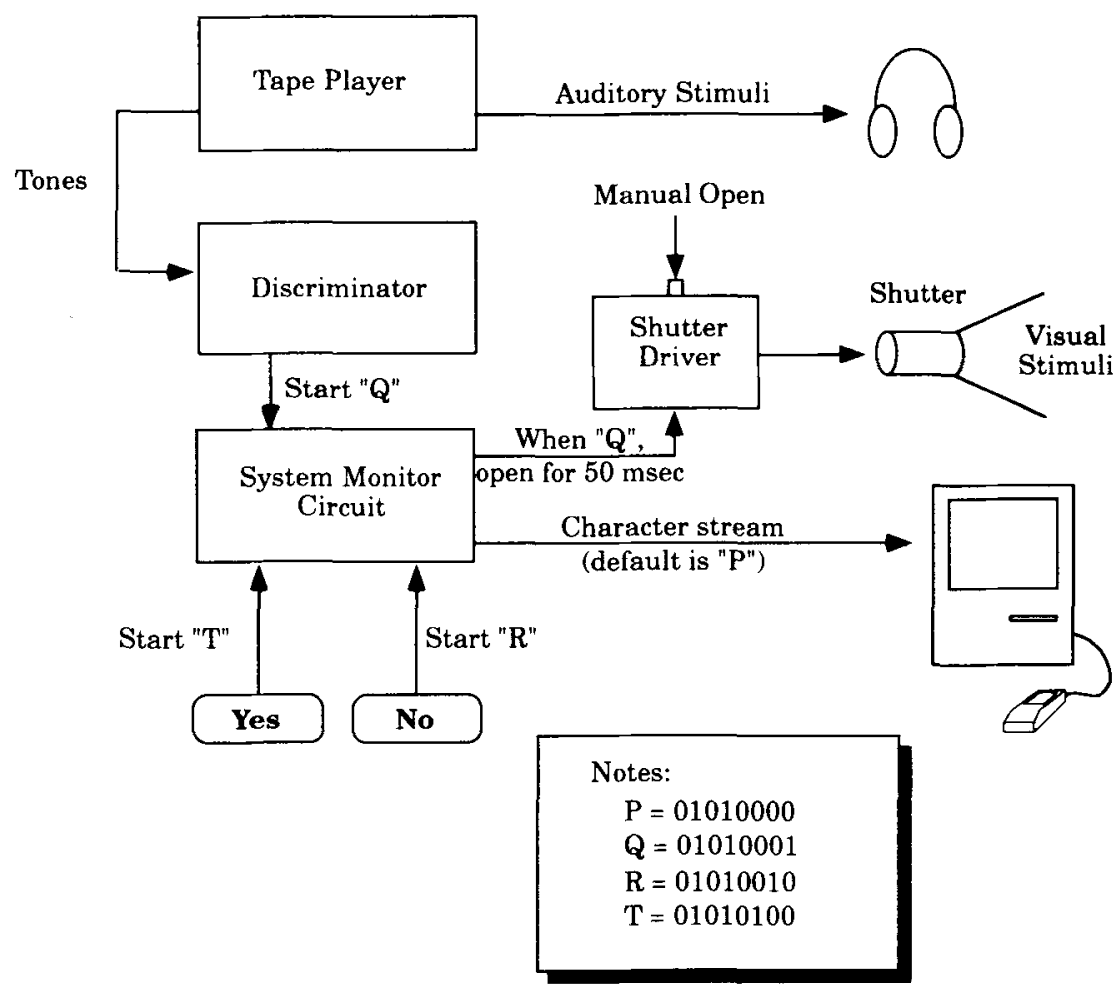

Figure 1. An overview, including connections, for setting up a cross-modal lexical priming experiment with the circuits and program described in this paper. 
that monitors the serial port for incoming information and calculates responses and reaction times on the basis of this information.

\section{Discriminator Circuit}

The circuit diagram for the discriminator circuit is depicted in Appendix A. As described above, this circuit detects the presence of a tone coming from an audio input. It filters extraneous pops and clicks (events with highfrequency components) from the input by giving a highfrequency rolloff at $500 \mathrm{~Hz}$. The audio signal can come either from one channel of a tape player or from the audio output jack on the Macintosh. The first op-amp (U1) amplifies the input signal by a factor of nine. Op-amps $\mathrm{U} 2$ and $\mathrm{U} 3$ form an absolute value circuit - they convert the ac signal into dc. U4 is a buffer amplifier that drives the $500-\mathrm{Hz}$ low-pass filter described above. The sensitivity of the discriminator circuit is controlled by adjusting the variable resistor connected to the inverting (-) input to the LM 311 just before the TTL output. As mentioned above, this feature allows the user to set the threshold so that the discriminator does not respond to extraneous signals.

\section{Shutter Driver}

The circuit diagram for the shutter driver is also depicted in Appendix A. A manual open is included to help with debugging during the experiment. The default setting is with the shutter closed. The purpose of the shutter driver is to keep the shutter open for the brief periods (50 msec for cross-modal priming studies) needed to display the visual stimuli. When the control voltage is high, the ITT3904 transistor is saturated. This turns off the SPS5025 Darlington pair, which turns off the current to the shutter, thereby closing it. The shutter opens when the control voltage is low. Manually closing the switch forces the control voltage low, allowing the shutter to be held open.

\section{System Monitoring Circuit}

The circuit diagram for this part of the configuration is depicted in detail in Appendix B. As noted above, the use of this circuit with the previously described discriminator circuit can allow any application to have external timing for reactions. Input to this circuit is from the two response keys and the discriminator circuit. The discriminator sets flip-flop $\mathrm{IC}_{4}$ and starts $\mathrm{IC}_{1}$, a 50-msec-width one-shot that opens the shutter. One-shots $\mathrm{IC}_{2}$ and $\mathrm{IC}_{3}$ are $O R$ 'd to reset the flip-flop, and their signals are also passed to the 4-bit $\mathrm{D}$ register $\mathrm{IC}_{5}$.

The purpose of the $\mathrm{D}$ register is to make sure that none of the bits change while being transmitted. Between the stop bit of one character and the start bit of the next, $\mathrm{IC}_{6}$ (a 40-nsec-width one-shot) enables the $\mathrm{D}$ register so that the four inputs pass through. The outputs are locked in while the character is being sent. $\mathrm{IC}_{7}$ and $\mathrm{IC}_{8}$, a 74150 multiplexer and 74163 counter, convert the parallel input from the $\mathrm{D}$ register into serial data (note that there are seven bits available for input and that this circuit uses only three; the rest are available for other uses). The $9600-$ $\mathrm{Hz}$ clock driving the counter is a 555 timer chip. The 7408 AND gate after the multiplexer, in combination with the External Enable Switch, allows the serial data stream to be turned off. Output goes to the shutter box and the RS232 serial port on the Macintosh. The output that is read by the serial port on the Macintosh is in the form of characters. " $P$ " is sent when nothing is happening (the default character), " $Q$ " is sent when the discriminator detects a tone (and the shutter opens). The characters " $R$ " and ' $T$ " 'are sent when the YES or NO switches, respectively, are closed. If both the YES and NO switches are hit simultaneously (within $5-10 \mathrm{msec}$ ), some of the characters sent will be " $X$ '"s. This signals an error condition.

The voltage sent to the shutter box is low for $50 \mathrm{msec}$, which opens the shutter for that duration. The voltage then goes high, closing the shutter.

\section{IMPLEMENTATION}

The system is hooked together with an audio cable from the sound source (the left or right channel of a tape deck or, perhaps, the sound port on the back of the Macintosh) to the discriminator, and an RS-232 to Macintosh cable which is hooked into the serial port of the Macintosh. Because the circuit responds to auditory events, the trigger can be a tone placed on a tape, or, if you are monitoring the Macintosh, a beep sound that is synchronized with the presentation of some stimulus. Note that if you are using a system beep (SysBeep), the synchronization of the visual stimulus and the beep is dependent on the frequency of the beep used. For example, the default beep sound on the Macintosh has a frequency around $1000-\mathrm{Hz}$, which makes synchronization accurate to $\pm 1 \mathrm{msec}$. Lower frequency tones would be slightly less accurate.

For those interested in conducting cross-modal lexical priming experiments, the shutter driver is connected to a tachistoscopic shutter via a connector that depends on the type of shutter used. Because the Lafayette shutter Model 5-06226 takes 30 $22 \mathrm{msec}$ to respond, the click must begin $30 \mathrm{msec}$ before the visual stimuli are to be displayed with this shutter. However, the character sending circuit can be used without the shutter if you simply wish to collect reaction times.

Figure 2 is a flowchart for a program, written and compiled in Symantec's THINK C, that monitors the serial port for incoming characters and does the necessary translation from number of characters received to reaction times. ${ }^{4}$ What follows is a brief description of the logic of the program as it is outlined in the figure.

Before data collection can begin, the program must allocate memory for the RESULT array into which all decisions and reaction times (RTs) will be recorded. Next, the serial port must be initialized and opened. Because this involves many Apple Toolbox calls, the necessary code is given in Appendix $\mathrm{C}$, along with the command used later 


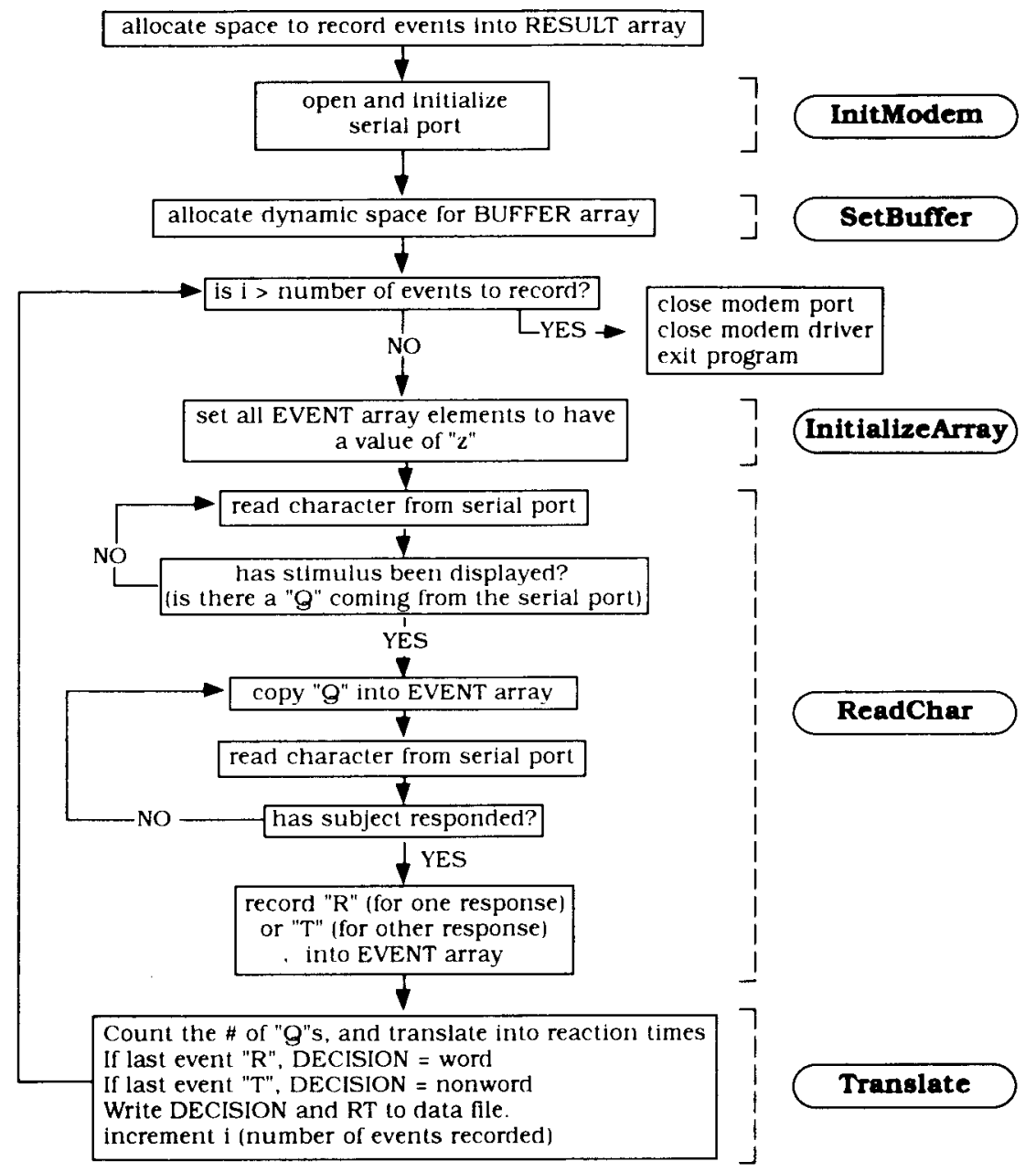

Figure 2. A flowchart of the code for collecting and recording reaction times on the Macintosh.

to fetch characters from the serial port (SerGetBuf). The program then dynamically allocates space for the serial buffer. This is necessary because the buffer normally allocates only 64 bytes to serial port information. After all the initializing is done, the system is ready to record reaction times. The logic for this part of the program is depicted in the parts of the flowchart that are labeled InitializeArray, ReadChar, and Translate. In brief, the circuit waits for the stimulus to be presented. Once presented, the circuit begins gathering characters (" $Q$ "'s, to be exact), awaiting a response from the subject. After the subject responds, the reaction time is calculated according to the following formula:

$$
\text { RT }=\frac{\text { NumberOfBits } \cdot 1000 \cdot \# " \text { "Q's }}{\text { BaudRate }},
$$

where NumberOfBits for our circuit is 10 , “ 'Q'"s is the number of characters received by the serial port between presentation of the stimulus and decision by the subject, and the BaudRate (as described earlier) is 970 characters per second. This reaction time is recorded, along with the decision made by the subject (as indicated by an " $R$ " or a " $T$ " character from the serial port), into a data file.

\section{FINAL COMMENTS}

The circuit and code described above allow researchers to record reaction times to externally presented stimuli with millisecond accuracy on a Macintosh computer. The apparatus described in this paper has been used in crossmodal lexical priming experiments, although, as described, the millisecond timer can be adapted for use in other paradigms.

\section{REFERENCES}

Inside Macintosh: Volume 1. (1988). New York: Addison-Wesley. RESNIK, R. A. (1990). Toolbox-based routines for Macintosh timing and display. Behavior Research Methods, Instruments, \& Computers, 22, 105-117.

SWINNEY, D. A. (1979). Lexical access during sentence comprehension: (Re)consideration of context effects. Journal of Verbal Learning \& Verbal Behavior, 14, 645-660. 
UlRICH, R., \& GiRAY, M. (1989). Time resolution of clocks: Effects on reaction time measurement-good news for bad clocks. British Journal of Mathematical \& Statistical Psychology, 42, 1-12.

Westall, R. [F.], Perkey, M. N., \& Chute, D. L. (1986). Accurate millisecond timing on Apple's Macintosh using Drexel's MilliTimer. Behavior Research Methods, Instruments, \& Computers, 18, 307-311.

Westall, R. F., Perkey, M. N., \& Chute, D. L. (1989). Millisecond timing on the Apple Macintosh: Updating Drexel's MilliTimer. Behavior Research Methods, Instruments, \& Computers, 21, 540-547.

ZWITSERLOOD, P. (1989). The locus of the effects of sentential-semantic context in spoken word processing. Cognition, 32, 25-64.

\section{NOTES}

1. The tachistoscopic shutter is a Lafayette shutter Model 5-06226, which can be purchased from Lafayette Instrument Co., P.O. Box 5729,
Lafayette, IN 47903 (phone: [301] 423-1505). This shutter responds $30 \pm 2 \mathrm{msec}$ after a signal from the shutter driver is detected.

2. A 50-msec presentation time for written words seems to give the most reliable cross-modal lexical priming effects (see, e.g., Zwitserlood, 1989).

3. These error estimates include both measurement and oscillator drift. The millisecond timer to measure the number of characters per second that were generated by our circuit was similar to that described in Westall et al. (1989). We recommend, however, counting the number of characters the serial port receives from the circuit while the Event Manager counts 600 ticks (approximately $10 \mathrm{sec}$ ), and calculating baud rate by dividing the number of characters received by $(16.67 \cdot 600)$. The error rate reported here $( \pm 2$ characters per second) reflects the accuracy of the millisecond timer in the circuit.

4. A copy of the complete code (in text format) is available from the first author or from the ftp site listed in the authors' notes.

\section{APPENDIX A}

Circuit Diagrams for the Discriminator Circuit and the Shutter Driver

\section{Discriminator Circuit}

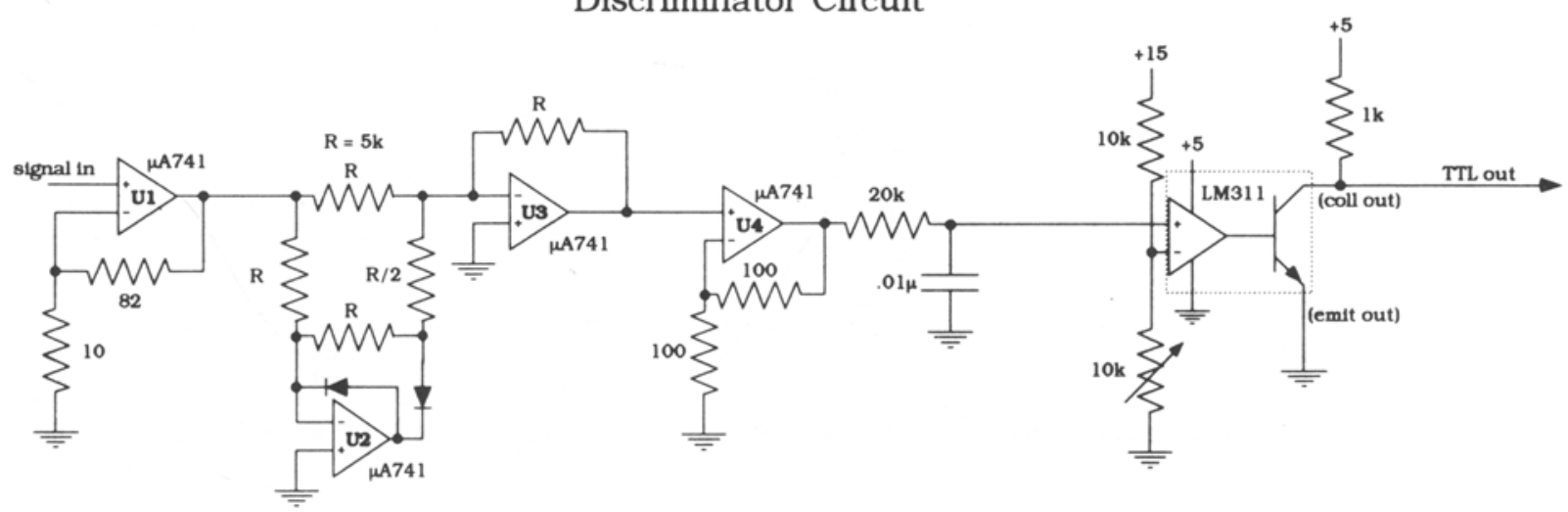

\section{Shutter Driver}

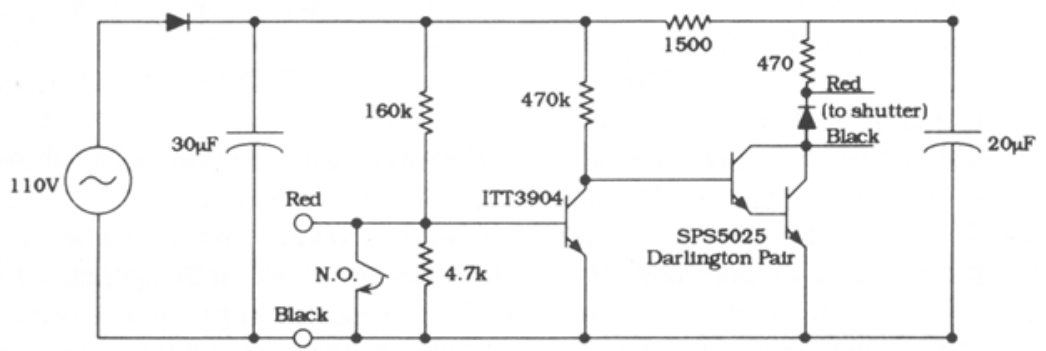

(to control voltage) 


\section{APPENDIX B}

Circuit Diagram for the System Monitor Circuit

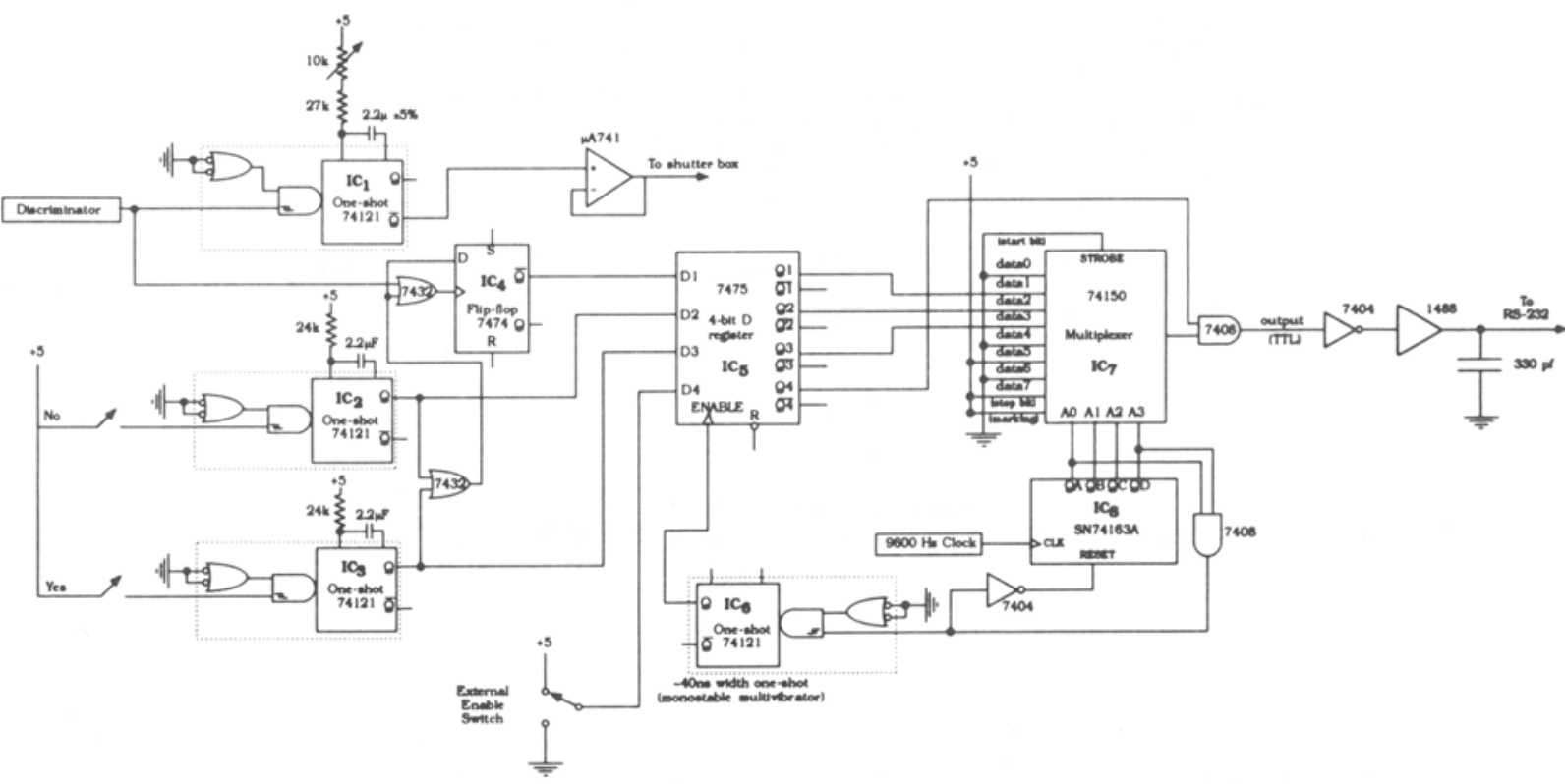

APPENDIX C

Apple Toolbox Commands for Initializing and Setting the Attributes of the Serial Port So That the Macintosh Can Interface With the Circuit Depicted in Appendix B

$\begin{array}{llll}\text { \#define } & \text { AscCR } & 13 & /^{*} \text { ASCII value of a carriage return } \\ \text { \#define } & \text { AscXOn } & 17 & /^{*} \text { ASCII for DCI (Control-Q) } \\ \text { \#define } & \text { AscXOff } & 19 & /^{*} \text { ASCII for DC3 (Control-S) }\end{array}$

$I^{*}$ Open the modem port for input, modemInptr refers to the driver reference number for the modem port, this value is used elsewhere in the program $*$ /

initmodem(modemInptr)

int ${ }^{*}$ modemlnptr;

\{SerShk handshake; $/^{*}$ HANDSHAKE is a variable: type SerShk */

$1^{*}$ RAMSDOpen opens the specified port-- sPortA refers to the modem port *

if (RAMSDOpen(sPortA) != noErr) /* For compatibility w/ older Macs */ printf "Cannot open RAM driver $\backslash n$ "); printf(" after RAMSDOpen\n");

$1^{*}$ OpenDriver opens the device driver specified in the first part (Ain) and returns its reference number in * modemInptr. Ain is for input throughthe modem port, this function returns the device driver name in modeminptr */

if (OpenDriver("\p.AIn", modemInptr) ! noErr) printf(" after OpenDriverไn");

$f^{*}$ SerReset resets and initializes the modemInptr driver according the the configuration in the second part, baud $9600+\ldots)^{*} /$

if (SerReset(*modemInptr, baud9600 + stop10 + noParity + data 7) != noErr) printf("Cannot set baud/data format $\backslash n$ ");

printf(" after SerReset\n");

$f^{*}$ the variable handshake is of the type SerShk which is a structure containing the following variable values (all chars) */
handshake.fXOn
$=0 ;$
$=0$
$l^{*}$ No outpul flow control */
$=0$
* CTS hardware handshake DISabled */

handshake.xOff

= AscXOn; $\quad{ }^{*}$ Xon character * 


\section{Appendix C (Continued)}

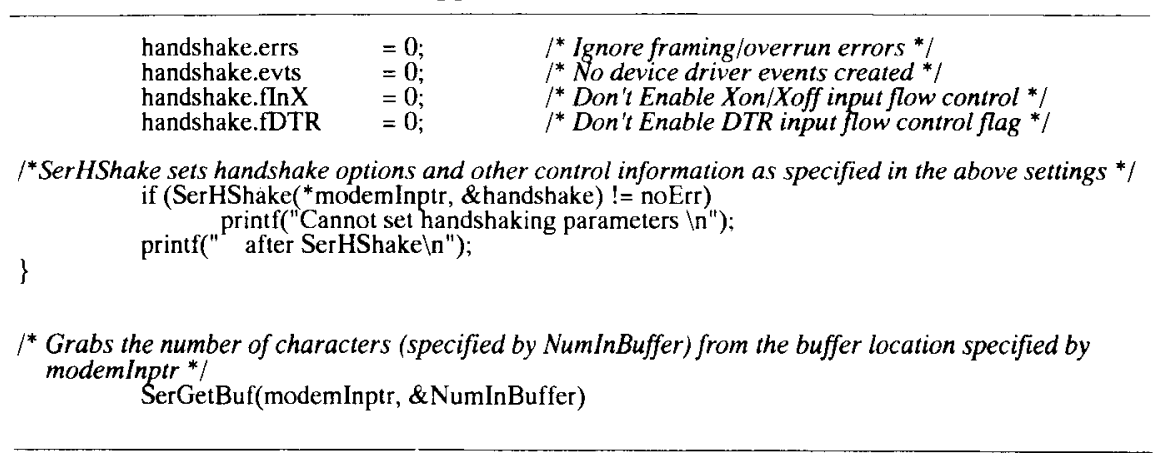

(Manuscript received July 31, 1991;

revision accepted for publication April 8, 1992.) 